\title{
Spinel ferrite/MnO core/shell nanoparticles: Chemical synthesis of all-oxide exchange biased architectures
}

Ombretta Masala, and Ram Seshadri

Materials Department and Materials Research Laboratory

University of California, Santa Barbara CA 93106 USA

masala@engineering.ucsb.edu, seshadri@mrl.ucsb.edu Fax: (805) 8938797

Supplementary figure

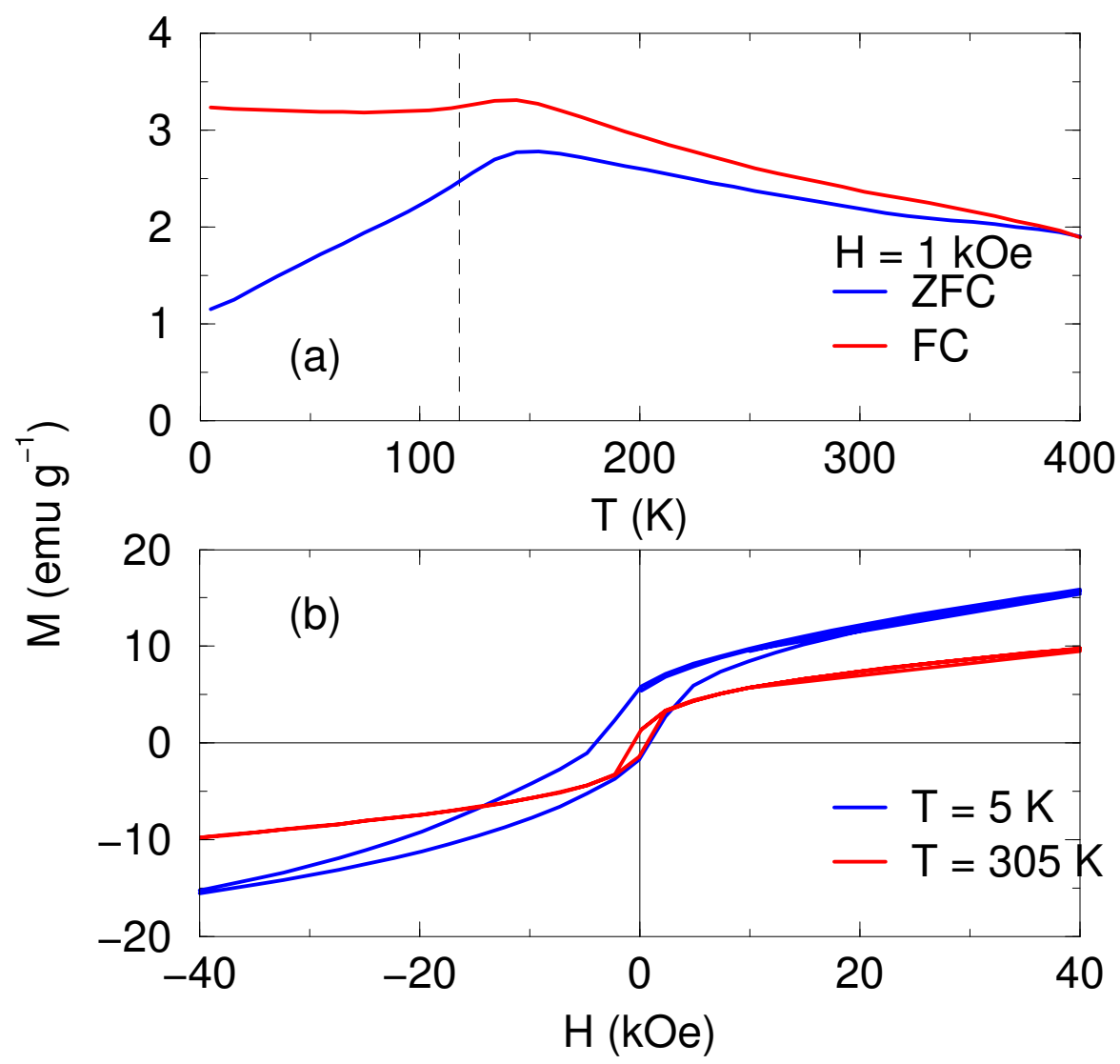

Supplementary figure: Magnetization as a function of temperature (a) and field (b) for a core/shell $\mathrm{ZnFe}_{2} \mathrm{O}_{4} / \mathrm{MnO}$ architecture. At $5 \mathrm{~K}$, the hysteresis loop displays some evidence for exchange biasing. The magnetization at $5 \mathrm{~K}$ and $40 \mathrm{kOe}$ is too large, by a factor of about 5 , for it to arise from uncompensated spins on nanoantiferromagnetic $\mathrm{MnO}$. 